\title{
A Lightweight Modular 12-DOF Print-and-Fold Hexapod
}

\author{
Daniel E. Soltero
}

Brian J. Julian
Cagdas D. Onal

Daniela Rus

\begin{abstract}
In this paper we present the design, fabrication and operation of a hexapod fabricated using a combination of printing and folding flat sheets of polyester. The polyester sheets are cut and engraved with crease patterns, which are then manually folded to create 3D functional modules, inspired by the Japanese art of Origami. These modules, when connected, form a hexapod with two degrees of freedom per leg. All custom mechanical parts are manufactured in a planar fashion using a laser cutter. We created this print-and-fold hexapod as a miniature version of a commercially available platform, to which we compare several metrics such as weight, walking speed, and cost of transportation. Our printed hexapod weights $195 \mathrm{~g}$, can walk at speeds of up to $38.1 \mathrm{~cm} / \mathrm{sec}$ (two body lengths per second) and can be manufactured and assembled from scratch by one person in approximately seven hours. Experimental results of gait control and trajectory tracking are provided.
\end{abstract}

\section{INTRODUCTION}

Imagine if we could build a customized body for a robotic platform only using commonly available materials like polyester sheets and acrylic, and a common laser cutter. Further imagine if you could share your robot body design for anyone in the community to replicate using common planar fabrication techniques. In this paper, we describe the design, analysis and fabrication of a lightweight hexapod robot using a combination of printing and folding flat sheets of polyester. By printing, we mean using a laser cutter to engrave crease patterns and cut boundaries in the polyester sheets. By folding, we mean transforming the 2D sheets into 3D functional modules (inspired by the Japanese art of Origami) with degrees of freedom (DOF) that can be actuated. Robots created with such technology were initially explored in [1], and we refer to them as print-and-fold robots.

Print-and-fold robot bodies do not require complex machining; all the customization is embedded in their 2D crease and cut patterns. This makes the print-and-fold technology an option for creating functional robotic platforms that require less time, effort and money to fabricate, compared to conventional robot fabrication methods. Additionally, it provides simple and fast means of customization through changes in the 2D crease and cut patterns. Since many planar substrate fabrication methods have been developed by the micro-fabrication industry (which can be used to create patterns in sheets of different materials [2]), creating any 2D crease pattern on a wide range of materials has become a tractable task.

This work was funded in part by NSF grants 1240383 and 1138967, \& the NSF Graduate Research Fellowship Award 0645960. We are grateful.

D. E. Soltero, B. J. Julian and D. Rus are with the Computer Science and Artificial Intelligence Laboratory, Massachusetts Institute of Technology, Cambridge, MA (soltero@mit.edu, bjulianecsail.mit.edu, rusecsail.mit.edu). C. D. Onal is with the Mechanical Engineering Department, Worcester Polytechnic Institute, Worcester, MA (cdonale wpi.edu).

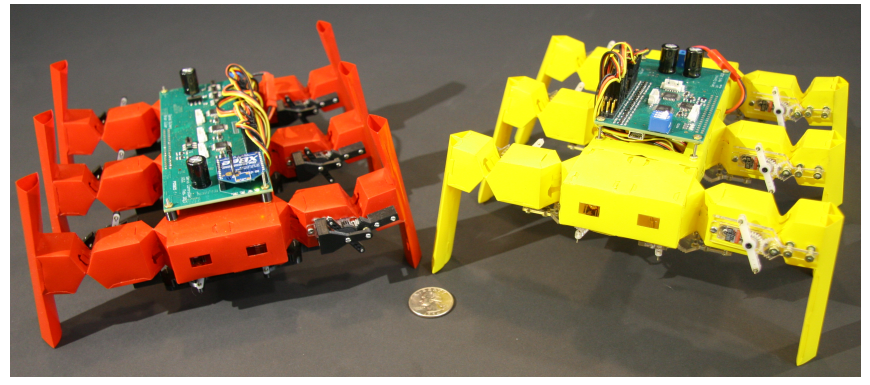

Fig. 1: Red and yellow FireAnt print-and-fold hexapods (with a US quarter for size reference).

In previous work [1], [3], we started exploring the capabilities of 3D actuated robotic structures folded out of 2D crease patterns on flat plastic sheets. This previous work showed how one could use Origami-inspired folding patterns to achieve locomotion. With the print-and-fold technology, [3] created a worm robot, [4] created a primitive hexapod, and [5] created a self-folding inchworm, demonstrating the possibility to ship robots as flat sheets to some destination and have them self-fold when the destination is reached. These prior print-and-fold robots were either actuated by Nickel Titanium coils (which are too inefficient and slow for our application) or by a small number of (1-2) motors. While simple to control, these robots had limited capabilities due to their limited DOF.

In this paper, we present a significant improvement to the capabilities of print-and-fold robots, by creating a printand-fold hexapod with two DOF per leg. All 12 DOF are individually actuated by commercially available servomotors, which enable more useful behaviors, such as accurate trajectory tracking and a variety of locomotion gaits. This paper provides experimental results of these behaviors. Our hexapod, like most print-and-fold robots, has the characteristic feature of being very lightweight since its body is primarily made of hollow polyhedra. The rigidity or stiffness in the robot mainly comes from the geometry of the folds, much like how channel brackets get their strength from bent sheet metal. The contributions of this paper are:

(i) a lightweight, rapidly fabricated print-and-fold hexapod, called the FireAnt, whose design can be open-sourced and easily customized,

(ii) the comparison between the FireAnt and a commercially available hexapod along several metrics,

(iii) the evaluation of the locomotion capabilities of the FireAnt.

The FireAnt was designed as a simplified, lighter and miniature version of a commercially available platform called APOD (Lynxmotion [6]). We compare the overall perfor- 
mances of the FireAnt and APOD to show that our printand-fold robot can perform as a miniature version of the commercially available platform, for a fraction of the weight and cost.

Many hexapod platforms have been built throughout the years, and many problems concerning hexapod locomotion have been studied. For example, [7] treats the problem of adaptive locomotion to achieve appropriate support on a hexapod robot, [8] treats the problem of unexpected rough terrain using biologically inspired reflexes in the legs of a robotic hexapod, and [9] shows how neural networks can be used to control the locomotion of a hexapod. More recent work on hexapods includes [10], where the design and control of a compliant-legged hexapod robot with one DOF per leg is described, and [11], where a decentralized controller for each leg of a hexapod was explored.

Related work on the idea of printing robots includes [12], where a process called smart composite microstructure (SCM) is used to create a hexapod robot by assembling 2D printed parts. The process consists of creating $2 \mathrm{D}$ robotic parts by bonding laser micro-machined composite fiber with polymer films. The final robot can be assembled by connecting together various parts of this kind. This process can be time consuming, although [12] showed that, using other materials, fabrication can be faster. In this paper, we describe a highly customizable robot that can be rapidly manufactured using printing and folding. Our solution is similar to the SCM process in that we laser cut and engrave 2D components, but our method does not rely on the use of rigid material (like composite fiber) for a rigid robotic structure. Our robots are made out of folded flexible polyester. Additionally, our method can be easily employed by the general public ${ }^{1}$ and it does not require a high level of precision as some SCM processes. ${ }^{2}$ The SCM process has been mainly used for the creation of very small-scale legged robots [13] and fliers [14], generating very promising results for such small scales. However, current robots using this technology have very simple mechanisms for locomotion, which is limiting for more complicated tasks.

\section{Print-And-Fold HeXAPod Design}

Figure 1 shows two 12-DOF print-and-fold hexapods called FireAnt hexapods: the original red prototype (hence the name FireAnt), and the latest design colored in yellow, which is the focus of this paper. The FireAnt, shown in more detail in Figure 2, is made out of spray-painted $0.127 \mathrm{~mm}$-thick flat sheets of moisture-resistant polyester (polyethylene terephthalate, commonly known as PET or PETE). It is an autonomous robotic hexapod with on-board power, computation and wireless communication. Each leg has two DOF which are independently actuated by servomotors. The control circuit is a custom printed circuit board which includes servomotor drivers, a low-level gait

\footnotetext{
${ }^{1}$ A laser cutter is needed, which can be "rented".

${ }^{2}$ In some SCM processes, the components must be aligned properly in a laser cutter, otherwise the parts will not be properly cut. In our method, there is no need for precision alignment of multiple parts.
}

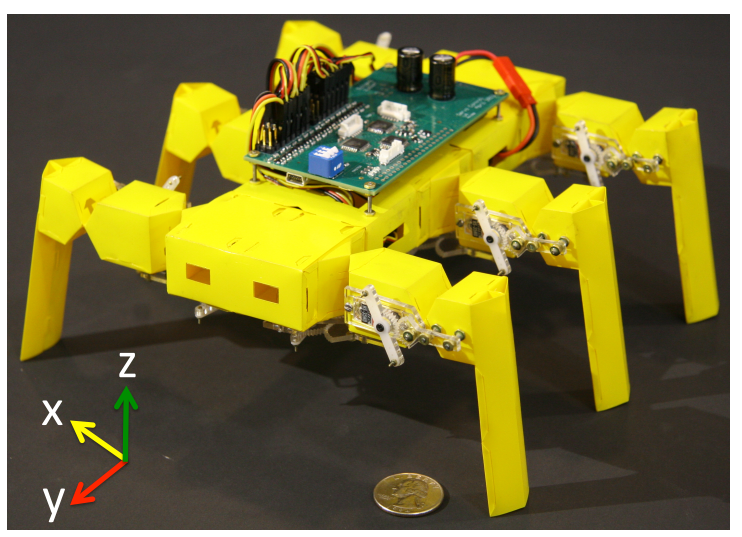

Fig. 2: A print-and-fold hexapod with two-DOF legs called FireAnt (with a US quarter for size reference).

coordinator and a Gumstix Overo Computer-On-Module interface for high-level control. Since each DOF in the legs is independently actuated, this robot is capable of executing an array of locomotion gaits. To date, we have successfully implemented the tripod gait, the ripple gait and the wave gait. Also, we have implemented variations of the tripod gait in order for the FireAnt to rotate and walk with a curvature radius. With these gaits, the FireAnt is capable of accurately tracking trajectories, as will be seen in Section IV.

The FireAnt design is modular and consists of seven 3D modules. A module is defined as a structure folded from a single 2D flat polyester sheet. Each module has a crease pattern engraved and boundaries cut by a laser cutter. Six of the FireAnt's seven modules correspond to the six legs, and the seventh module corresponds to the robot's body, which holds the leg modules together to form the hexapod. Although the full robot can be folded from a single sheet, our proposed modular design is more robust and allows the user to swap components to replace damaged parts or test alternative designs. Additionally, we found empirically that the modular design gives the FireAnt a rigid body without the need of adding complicated geometric folds in the body to provide stiffness.

A key feature of the FireAnt hexapod is that all custom mechanical parts are manufactured in a planar fashion using a laser cutter. This makes the FireAnt hexapod very easy and fast to manufacture.

\section{A. Leg Modules}

Leg modules for the FireAnt are folded from the 2D crease/cut pattern shown in Figure 3. The solid lines represent cuts performed by the laser cutter (either boundaries of the module or holes within the module), and the dashed lines represent engraved creases to perform folds. This pattern includes holes and openings for the two servomotors that actuate the two movable joints in the leg. These two joints are orthogonal to each other and correspond to a horizontal hip rotation to swing the leg back and forth, and a vertical knee rotation to lift and lower the leg.

Many robots use direct drive actuators, where a motor's location defines a joint's location, and the post-joint segment 


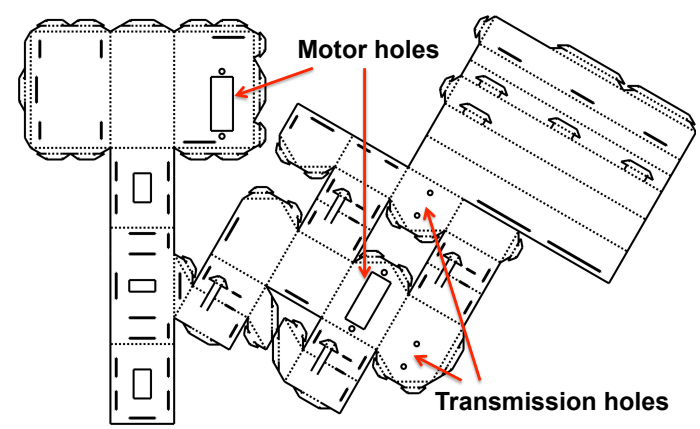

Fig. 3: The 2D crease/cut pattern for the leg modules. Solid lines represent cuts and dashed lines represent creases.

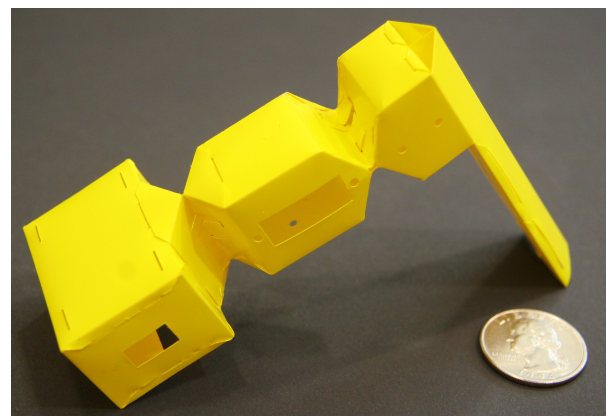

Fig. 4: A folded 3D leg module with two orthogonal DOF (with a US quarter for size reference).

is attached to the motor horn. However, for print-and-fold robot legs made from a single sheet (as is the case for the leg modules), it is very hard to install motors exactly at the joint's center of rotation. ${ }^{3}$ Therefore, a transmission gearbox is needed to transmit the torque from the servomotor's center of rotation to the joint's center of rotation. The transmission used in the FireAnt consists of a 1:1 gearbox made out of laser-cut acrylic (hence it can be manufactures quickly and easily). The crease/cut pattern in Figure 3 includes holes to hold the servomotors and their transmissions with screws.

Once the 2D crease/cut pattern from Figure 3 is engraved/cut on a flat polyester sheet, we can fold the sheet into the 3D leg module seen in Figure 4. Adding the two servomotors and the transmission gears completes the leg module. Each leg module's crease/cut pattern requires approximately 10 minutes to be engraved/cut by a VersaLASER laser cutter, and 53 minutes for a single person to manually fold it into its 3D structure (with motors and transmissions installed).

\section{B. Body Module}

The 2D crease/cut pattern for this module can be seen in Figure 5. The main purpose of this module is to hold the six leg modules in place. It does this by the use of small tabs that grab and hold on to the leg modules in different locations. The 3D body module, folded from this crease/cut pattern, can be seen in Figure 6. The two small box-like compartments seen in this figure can be used to store batteries and cables. The body module crease/cut pattern requires approximately

\footnotetext{
${ }^{3}$ If, for example, two sheets of polyester are used, then one sheet can hold on to the motor and the other sheet can hold on to the motor horn.
}

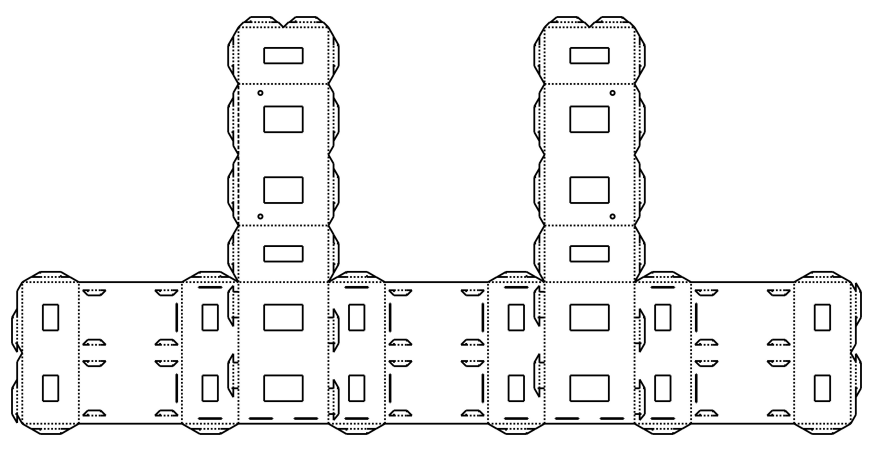

Fig. 5: The 2D crease/cut pattern for the body module. Solid lines represent cuts and dashed lines represent creases.

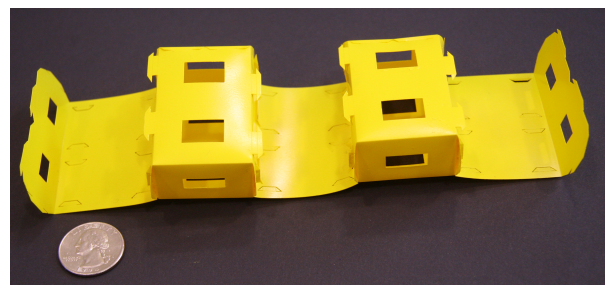

Fig. 6: A folded 3D body module (with a US quarter for size reference). This module includes tabs to grab and hold on to the leg modules.

15 minutes to be engraved/cut by a VersaLASER laser cutter, and 12 minutes to be manually folded into its 3D structure.

Once six leg modules and the body module are folded, we assemble the final hexapod by connecting all the modules. This process requires approximately 75 minutes to be completed. After adding a control circuit and a battery, the final print-and-fold FireAnt platform can be seen in Figure 2. Assuming all materials/components are readily available, the FireAnt hexapod can be manufactured and assembled from scratch by a single person in approximately 7 hours.

\section{PlatForm ANALYSis}

In this section we consider the kinematic capabilities and the physical specifications of the FireAnt. Note that, for simplicity, we assume the FireAnt behaves like a rigid-body robot.

\section{A. Kinematics}

Figure 7 shows the standard rigid-body kinematic analysis diagram of one of the FireAnt's leg modules. In this figure, the hip location corresponds to the origin of our analysis, where the Euclidean $(x, z)$ axes are shown. The red dot at the origin represents the hip joint, which can rotate along the transverse axis. The second red dot, separated from the origin by the femur (of length $L_{f}$ ), represents the knee joint, which can rotate along the coronal axis. The third red dot, at the bottom of Figure 7 represents the end effector, which is separated from the knew joint by the tibia (of length $L_{t}$ ) and an additional offset segment (of length $L_{o}$ ), which forms a right angle with the tibia.

Continuing the rigid-body kinematic analysis, we conclude that the Euclidean position of the leg's end effector (the edge of the leg which touches the floor), with respect to the hip 


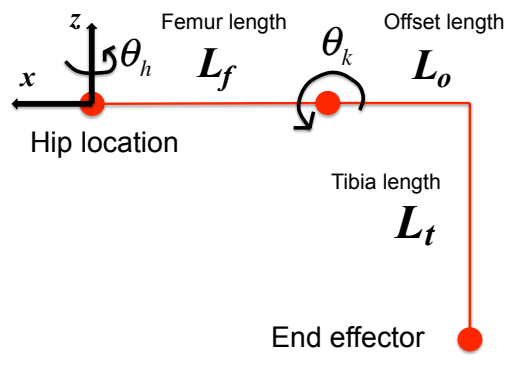

Fig. 7: Rigid-body kinematic analysis diagram of a leg module.

location, obeys the following equation:

$$
\left[\begin{array}{l}
x \\
y \\
z
\end{array}\right]=\left[\begin{array}{c}
-\cos \left(\theta_{h}\right)\left(L_{f}+L_{t} \sin \left(\theta_{k}\right)+L_{o} \cos \left(\theta_{k}\right)\right) \\
-\sin \left(\theta_{h}\right)\left(L_{f}+L_{t} \sin \left(\theta_{k}\right)+L_{o} \cos \left(\theta_{k}\right)\right) \\
L_{o} \sin \left(\theta_{k}\right)-L_{t} \cos \left(\theta_{k}\right)
\end{array}\right],
$$

where $\theta_{h}$ represents the hip-joint angle and $\theta_{k}$ represents the knee-joint angle. From the above equation, we see that the end effector's height (whether the leg is lifted or grounded) is controlled solely by the knee angle $\theta_{k}$. There is only one additional DOF to control both the $x$ and $y$ coordinates of the end effector. Therefore, we cannot place the end effector at arbitrary locations. This is computed as $x^{2}+y^{2}=\left(L_{f}+L_{t} \sin \left(\theta_{k}\right)+L_{o} \cos \left(\theta_{k}\right)\right)^{2}$. However, when the leg is grounded, i.e. $\theta_{k} \simeq 0$, assuming that $\theta_{h}$ is small, we have

$$
\begin{aligned}
& x \approx-\left(L_{f}+L_{t} \theta_{k}+L_{o}\right) \approx-\left(L_{f}+L_{o}\right), \\
& y \approx-\theta_{h}\left(L_{f}+L_{t} \theta_{k}+L_{o}\right) \approx-\theta_{h}\left(L_{f}+L_{o}\right) .
\end{aligned}
$$

So, if the hip angle is maintained small, the FireAnt can approximately walk forward (or backward) in a straight line.

\section{B. Motor Requirements}

We now calculate how much torque the servomotors need to generate in order to support the weight of the FireAnt. We do this by performing a static force analysis and conservatively over-designing to get acceptable dynamics. To simplify the calculations, we assume the robot is static with $\theta_{h}=0$ and $\theta_{k}=0$ for all grounded legs. With this assumption, the worst case scenario is the hexapod holding itself up with only three legs, like it would do while performing a tripod gait. We modeled the robot's mass as a single point at the robot's center of gravity (CG), which is located at the geometric center of the robot. A diagram depicting this analysis from a top view can be seen in Figure 8, where the three red dots represent the locations of the three supporting legs. Each supporting leg is associated with a ground force exerted on it and represented by $F_{i}, i=1,2,3$. The distances $D_{1}$ and $D_{2}$ are the distances from the center of gravity to the legs' contact points with the ground.

Performing a static sum of forces and sum of torques to maintain equilibrium, we obtain:

$$
\begin{array}{rlr}
\sum F_{i} & =F_{1}+F_{2}+F_{3} & =M g, \\
\sum M_{x} & =D_{2} F_{1}-D_{2} F_{3} & =0, \\
\sum M_{y} & =D_{1} F_{2}-D_{1} F_{1}-D_{1} F_{3} & =0,
\end{array}
$$

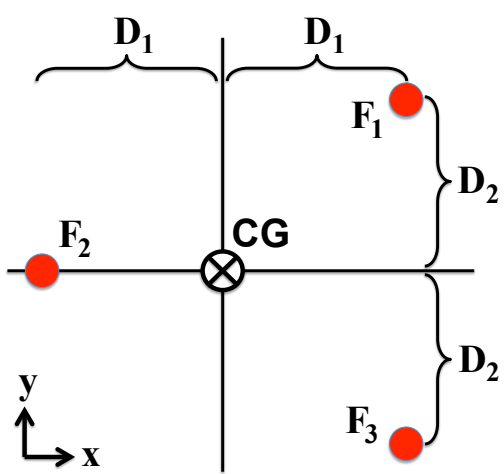

Fig. 8: Static force analysis diagram from a top view. CG represents the robot's center of gravity, located at the center of the robot's body. For this analysis, the robot is supporting itself with only three legs that are extended orthogonally from the body. The location of the three supporting legs are represented by the three red circles, each with an associated ground force being exerted on it and represented by $F_{i}, i=1,2,3$.

where $M$ is the robot's mass and $g$ is the gravitational acceleration constant. From these three equations we obtain that $F_{1}=F_{3}=M g / 4$ and $F_{2}=M g / 2$. Hence, the middle leg has to support the most weight. From Figure 7, a force of $F_{2}=M g / 2$ pushing upward in the end effector translates into a torque at the knee joint of $L_{o} M g / 2$. Since we intend to use the same motor for all joints, we require the motor torque specification $T_{m}$ to satisfy $T_{m}>L_{o} M g / 2$ in order for the robot to support itself.

For the FireAnt design, the offset length $L_{o}$ has a value of $2.3 \mathrm{~cm}$, and the robot's mass depends on the mass of the motors. So a trade-off between motor torque and mass must be reached in order to meet the requirement. We found that the Hitec HS-35HD servomotors produce a total robot mass of $195 \mathrm{~g}$, which gives us a required motor torque of $T_{m}>22.0 \mathrm{mN} \cdot \mathrm{m}$. The Hitec HS-35HD servomotors have a torque specification of $T_{m}=78.5 \mathrm{mN} \cdot \mathrm{m}$, which meets the requirement, and, after implementation, provided acceptable dynamics.

\section{EXPERIMENTS}

In this section we: 1) show how to control the FireAnt's 12 DOF in order to produce different locomotion gaits, 2) provide experimental results of the FireAnt's ability to accurately track trajectories in flat surfaces, and 3) compare the FireAnt to a commercially available platform according to several metrics. The FireAnt is controlled using a custom printed circuit board which includes drivers for each servomotor and a gait coordinator. This coordinator synchronizes the legs while they move in periodic pre-programmed sequences specific to the desired gait.

\section{A. Gait Coordinator}

We implemented three basic gaits for the FireAnt: tripod gait, ripple gait and wave gait. These gaits are well studied in the robotics and biomechanics literature, e.g. [15]. We use the following notation for the FireAnt legs: $L_{1}$ for front left, $L_{2}$ for middle left, $L_{3}$ for rear left, $R_{1}$ for front right, $R_{2}$ for middle right, and $R_{3}$ for rear right. 
The periodic pre-programmed leg sequences for the three basic gaits are described in Figure 9. This figure shows the command angles (in radians) over time sent by the gait coordinator to the servomotors for each leg's hip and knee joints. The variable period at which these periodic sequences are run defines the speed at which the robot travels. This speed is typically limited by the maximum speed at which the servomotors can move.

These gait sequences generated acceptable performance on different flat surfaces, including concrete, wood, nonslip PVC, carpet, and linoleum. To date, the FireAnt platform has traveled a total of more than 800 meters (4189 times its body length) using these gaits. The FireAnt achieved a maximum walking speed of approximately $38.1 \mathrm{~cm} / \mathrm{sec}$ (two body lengths per second) on the nonslip PVC surface using the tripod gait.

Figure 10 shows the distance that the FireAnt traveled through time in a single experiment with the pre-programmed tripod, ripple and wave gaits specified in Figure 9 on a flat linoleum surface. These results correspond to the preprogrammed periodic patterns for the three basic gaits using a constant loop rate, such that the magnitude of the slope in Figure 9 of the knee angle when a leg is not grounded is approximately $2 \pi / \mathrm{sec}$. This generates a period of 0.6 seconds for the tripod gait sequence, 0.9 seconds for the ripple gait sequence and 1.8 seconds for the wave gait sequence.

The FireAnt uses a $250 \mathrm{~mA} \cdot \mathrm{h}$ 3-cell lithium polymer battery pack to power its components. It was allowed to walk straight forward until the battery was depleted using the tripod gait on a flat concrete surface at a speed of $9.4 \mathrm{~cm} / \mathrm{sec}$. This process was repeated three times, yielding a mean battery life of 39.1 minutes. This translates in the FireAnt consuming on average 4.3 watts of power and having a mean cost of transportation of $2.5 \mathrm{~mJ} / \mathrm{g} / \mathrm{cm}$ at the specified walking speed when using only the gait coordinator.

\section{B. Trajectory Tracking}

In addition to the three basic gaits described in Section IV-A, two variations of the tripod gait were implemented. Firstly, by reversing the direction of the hip actuation on one side of the hexapod during the tripod gait, the FireAnt can rotate. Secondly, while maintaining the shape of the tripod stance, but shortening inter-tripod distances in one of the robot's side, the FireAnt can walk forward (or backward) with a curvature [16].

With these two additional gaits, the FireAnt is capable of tracking a trajectory. To do this, the FireAnt's low-level gait coordinator described in Section IV-A interfaces with a Gumstix Overo Computer-On-Module (COM) running an Ubuntu operating system and the Robot Operating System (ROS) software framework. The Gumstix Overo COM runs the controller specified in Algorithm 1, which uses feedback to calculate the desired heading in order to reduce the tracking error. Lines 5 and 7 of Algorithm 1 correspond to the Gumstix Overo COM communicating with the lowlevel gait coordinator from Section IV-A to select the proper gait to reduce the tracking error. In our experiments, the
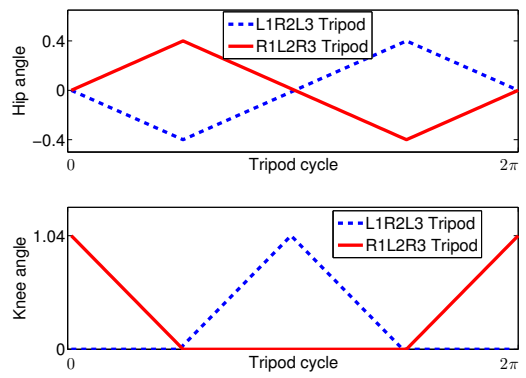

(a) Tripod gait periodic sequence
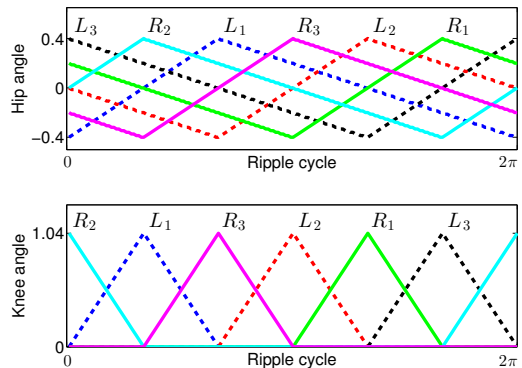

(b) Ripple gait periodic sequence
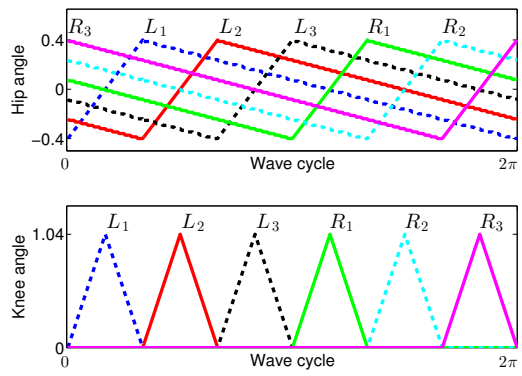

(c) Wave gait periodic sequence

Fig. 9: Periodic pre-programmed hip and knee sequences for the (a) tripod gait, (b) ripple gait, and (c) wave gait. Each sequence is the input in radians to the corresponding servomotor in order to generate the corresponding gait.

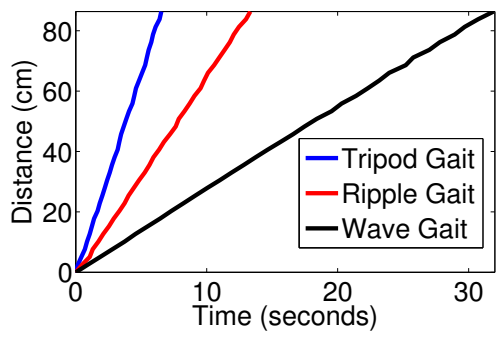

Fig. 10: The FireAnt's distance traveled through time using the tripod, ripple and wave periodic pre-programmed leg sequences. The data for this figure was obtained by parsing a video of the experiment.

Gumstix Overo COM receives global position information from a Vicon motion capture system in order to calculate the desired heading for the FireAnt, although, in the future, we wish to replace the motion capture system with a camera mounted on the robot to perform this task visually.

The FireAnt used this controller to track 15 random paths on a flat linoleum surface. Each path was generated by the 

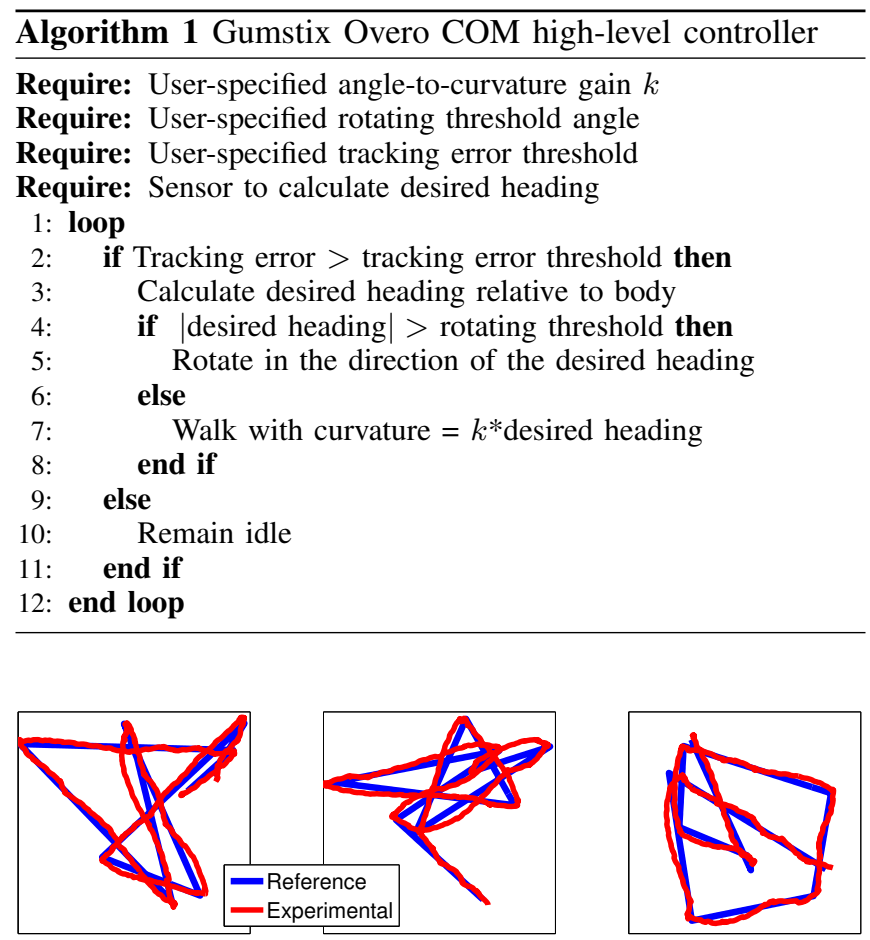

Fig. 11: Three of the 15 randomly generated paths (in blue) in a $150 \mathrm{~cm} \times 150 \mathrm{~cm}$ area, along with the FireAnt's experimental path (in red).

linear interpolation ${ }^{4}$ of 10 consecutive randomly-generated waypoints within a square area of $150 \mathrm{~cm} \times 150 \mathrm{~cm}$. Three of the 15 randomly generated paths can be seen as blue lines in Figure 11, along with the FireAnt's experimental path, shown in red.

We define the trajectory tracking error as the distance from the FireAnt's physical position to its nominal position if it followed the path perfectly at a constant speed of $6 \mathrm{~cm} / \mathrm{sec}$. The difference between the FireAnt's physical and nominal position determines the desired heading in Algorithm 1. The tracking error through time is shown in Figure 12, where the black line corresponds to the average of the 15 tracking errors over time, and the blue vertical lines represent the range of maximum to minimum tracking errors at the corresponding points in time. For a tracking speed of $6 \mathrm{~cm} / \mathrm{sec}$, the FireAnt tracked the random paths with an average error of under $20 \mathrm{~cm}$, although it obtained tracking errors of up to $42.2 \mathrm{~cm}$ ( 2.2 body lengths), mostly due to having to rotate in order to follow the sudden direction changes in the random paths. For a path with less abrupt direction changes, such as the one shown in Figure 13, the FireAnt performs significantly better (error has a bound of 0.7 body lengths). This can be seen in Figure 12, where the red line represents the tracking error for a single experiment of the FireAnt tracking the path shown in Figure 13.

\footnotetext{
${ }^{4}$ Linear interpolation is not necessary for path tracking; it was selected due to its simplicity. The FireAnt can track a path with curvature, like the one shown in Figure 13.
}

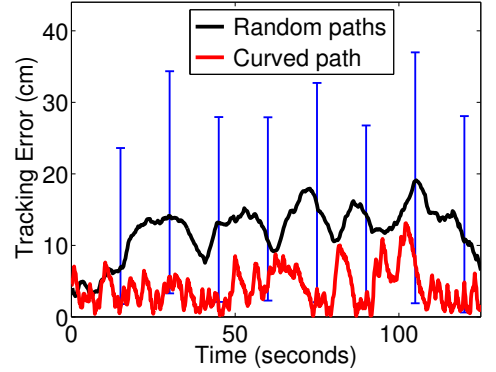

Fig. 12: Experimental tracking errors. The black line shows the average tracking error for the 15 random paths. The blue vertical lines represent the range of maximum to minimum errors through time for these paths. The red line represents the tracking error for the path shown in Figure 13.

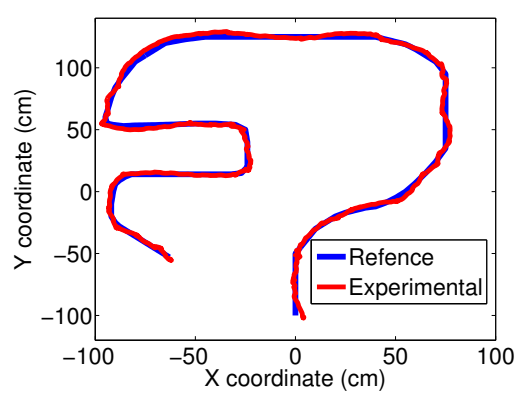

Fig. 13: Additional testing path (in blue) with no abrupt direction changes, along with the FireAnt's experimental path (in red).

\section{Comparison with a commercially available platform}

Next we compare the FireAnt to the commercially available platform called APOD [6]. Since we did not fabricate a head with a gripper or a tail for the FireAnt, the head and tail of the APOD platform were removed. All comparisons and measurements shown are with the head-less, tail-less APOD. Table I shows the parameters for both platforms for comparison. The FireAnt's dimensions can also be expressed as: $53 \%$ of the APOD's overall height, $46 \%$ of the APOD's overall width and $80 \%$ of the APOD's length. Most importantly, notice that FireAnt's mass is $8 \%$ of the APOD's mass.

When comparing several performance metrics between the APOD and the FireAnt, we obtain the values shown in Table II, where "BL" represents body length and "BW" represents body weight. The payload performance is an approximate value, which was obtained experimentally by placing additional weight on the robots until they couldn't walk with a tripod gait. ${ }^{5}$ The APOD cost of transportation was obtained for a walking speed of $25.4 \mathrm{~cm} / \mathrm{sec}$ on flat carpet, which yielded a battery life of approximately 30 minutes and a power consumption of 33.6 watts. From Table II, we can see that the FireAnt can walk faster than the APOD, although the APOD can carry a slightly larger relative payload than the FireAnt. Additionally, the FireAnt's cost of transport is higher than the APOD's, although its power consumption is lower, making the FireAnt a lowpower alternative.

\footnotetext{
${ }^{5} \mathrm{~A}$ point of failure for the FireAnt while carrying such payload was the breaking of servomotors' internal plastic gears.
} 
TABLE I: Table of Hexapod Parameters

\begin{tabular}{|l|l|l|}
\hline Parameter & APOD & FireAnt \\
\hline \# of servomotors & 18 & 12 \\
\# of legs & 6 & 6 \\
DOF per leg & 3 & 2 \\
Height (body) & $6.4 \mathrm{~cm}^{*}$ & $2.2 \mathrm{~cm}$ \\
Height (overall) & up to $21.6 \mathrm{~cm}^{*}$ & $11.4 \mathrm{~cm}$ \\
Width (body) & $16.8 \mathrm{~cm}^{*}$ & $5.5 \mathrm{~cm}$ \\
Width (overall) & $43.2 \mathrm{~cm}^{*}$ & $19.7 \mathrm{~cm}$ \\
Length & $23.8 \mathrm{~cm}^{*}$ & $19.1 \mathrm{~cm}$ \\
Ground clearance & up to $19.1 \mathrm{~cm}^{*}$ & up to $4.4 \mathrm{~cm}$ \\
Mass (with batteries) & $2369 \mathrm{~g}$ & $195 \mathrm{~g}$ \\
\hline
\end{tabular}

*Value was obtained from [6].

TABLE II: Performance Table

\begin{tabular}{|l|l|l|}
\hline Performance metric & APOD & FireAnt \\
\hline Maximum speed & $30.5 \mathrm{~cm} / \mathrm{sec}^{*}$ & $38.10 \mathrm{~cm} / \mathrm{sec}$ \\
Relative maximum speed & $1.3 \mathrm{BL} / \mathrm{sec}^{* *}$ & $2.0 \mathrm{BL} / \mathrm{sec}^{* *}$ \\
Payload & $3900 \mathrm{~g}$ & $265 \mathrm{~g}$ \\
Relative payload & $1.7 \mathrm{BW}{ }^{* *}$ & $1.4 \mathrm{BW} * *$ \\
Cost of transportation & $0.5 \mathrm{~mJ} / \mathrm{g} / \mathrm{cm}$ & $2.5 \mathrm{~mJ} / \mathrm{g} / \mathrm{cm}$ \\
\hline
\end{tabular}

*Obtained experimentally, although [6] states $25.4 \mathrm{~cm} / \mathrm{sec}$.

**BL refers to body length and BW refers to body weight.

\section{Final Remarks and Future Work}

With the print-and-fold robotics technology, the FireAnt design can be easily customized by editing the crease/cut patterns shown in Figures 3 and 5. Such changes can, for example: 1) add functionality by adding grippers, 2) change the size of the robot by scaling it, elongating the legs or widening the body, 3) make a quadruped robot by eliminating two legs, or 4) add more joints. If motors and circuitry are reused, the price for making such changes is negligible, since it falls under the cost of polyester sheets, which, for the FireAnt design, is $0.5 \%$ of the total price. Additionally, a full FireAnt robot can be manufactured and assembled from scratch by a single person in a very small amount of time (approximately 7 hours).

The folding of the FireAnt's 2D polyester sheets into the 3D modules is currently performed manually and requires some experience. In the future we wish to make the designs and folding process more intuitive and easier for nonexperienced users. Also, in this work we assume the robot has a rigid body, and we do not perform a deformability analysis on the print-and-fold elements. In the future, it would be useful to perform such an analysis, and design next generation print-and-fold robots based on such analyses.

\section{CONCLUSION}

We have presented the design, construction and operation of a fully autonomous print-and-fold hexapod with two DOF per leg. The robot is built out of six leg modules attached together by a body module, all folded from flat sheets of polyester with engraved crease patterns. Each leg module contains two servomotors that independently actuate the two DOF. We performed a rigid-body kinematic analysis of the platform and a static force analysis to calculate the motor requirements. We built our platform and implemented five gaits: tripod, ripple, wave, rotation, and tripod with curvature. Using the last two gaits, we showed that, if reliable desired heading information is available, the FireAnt is capable of tracking a trajectory reliably. To further demonstrate the utility of the print-and-fold robot, we compared it with a commercially available robot. Through empirical data, we showed that the FireAnt approximately performs as a very lightweight miniature version of the commercial platform.

The print-and-fold FireAnt hexapod offers the robotics field a viable option for an inexpensive, very lightweight and highly customizable hexapod robot that can be manufactured very quickly. Combined with the potential of self-folding robots, which are currently under investigation, print-andfold robots could become the new standard in robotics for tasks where having many inexpensive, lightweight and quickly-manufacturable robots is more valuable than a few very expensive heavy robots.

\section{REFERENCES}

[1] C. Onal, R. Wood, and D. Rus, "Towards printable robotics: Origamiinspired planar fabrication of three-dimensional mechanisms," in Robotics and Automation (ICRA), 2011 IEEE International Conference on, may 2011, pp. $4608-4613$.

[2] N. Bassik, G. M. Stern, and D. H. Gracias, "Microassembly based on hands free origami with bidirectional curvature," Applied Physics Letters, vol. 95, no. 9, pp. 091901-091 901, 2009.

[3] C. Onal, R. Wood, and D. Rus, "An origami-inspired approach to worm robots," Mechatronics, IEEE/ASME Transactions on, vol. 18, no. 2, pp. $430-438$, april 2013.

[4] C. D. Onal, M. T. Tolley, K. Koyanagi, R. J. Wood, and D. Rus, "Shape memory alloy actuation of a folded bio-inspired hexapod," in ATBio Workshop, IROS, 2012.

[5] S. M. Felton, M. T. Tolley, C. D. Onal, D. Rus, and R. J. Wood, "Robot self-assembly by folding: A printed inchworm robot," in Robotics and Automation (ICRA), 2013 IEEE International Conference on, 2013, to appear.

[6] [Online]. Available: http://www.lynxmotion.com

[7] R. B. McGhee and G. I. Iswandhi, "Adaptive locomotion of a multilegged robot over rough terrain," Systems, Man and Cybernetics, IEEE Transactions on, vol. 9, no. 4, pp. 176 -182, april 1979.

[8] K. S. Espenschied, R. D. Quinn, R. D. Beer, and H. J. Chiel, "Biologically based distributed control and local reflexes improve rough terrain locomotion in a hexapod robot," Robotics and Autonomous Systems, vol. 18 , pp. $59-64,1996$

[9] J. C. Gallagher, R. D. Beer, K. S. Espenschied, and R. D. Quinn, "Application of evolved locomotion controllers to a hexapod robot," Robotics and Autonomous Systems, vol. 19, no. 1, pp. 95 - 103, 1996.

[10] U. Saranli, M. Buehler, and D. E. Koditschek, "Rhex: A simple and highly mobile hexapod robot," The International Journal of Robotics Research, vol. 20, no. 7, pp. 616-631, 2001.

[11] T. Barfoot, E. Earon, and G. DłEleuterio, "Experiments in learning distributed control for a hexapod robot," Robotics and Autonomous Systems, vol. 54, no. 10, pp. $864-872,2006$.

[12] A. Hoover and R. Fearing, "Fast scale prototyping for folded millirobots," in Robotics and Automation, 2008. ICRA 2008. IEEE International Conference on, May, pp. 1777-1778.

[13] A. T. Baisch, C. Heimlich, M. Karpelson, and R. Wood, "Hamr3: An autonomous $1.7 \mathrm{~g}$ ambulatory robot," in Intelligent Robots and Systems (IROS), 2011 IEEE/RSJ International Conference on, Sept., pp. 50735079 .

[14] R. Wood, "The first takeoff of a biologically inspired at-scale robotic insect," Robotics, IEEE Transactions on, vol. 24, no. 2, pp. 341-347, April, 2008

[15] C. Ferrell, "A comparison of three insect-inspired locomotion controllers," Robotics and Autonomous Systems, vol. 16, pp. 135 - 159, 1995.

[16] C. Zollikofer, "Stepping patterns in ants-influence of speed and curvature," Journal of experimental biology, vol. 192, no. 1, pp. 95-106, 1994. 